\title{
CONCORDANCIA HISTOPATOLÓGICA ENTRE LA CITOLOGÍA Y LA BIOPSIA ANAL EN HOMBRES QUE TIENEN SEXO CON HOMBRES PORTADORES DE VIH Y NEOPLASIA INTRAEPITELIAL ANAL ASOCIADA A VPH
}

\author{
HISTOPATHOLOGICAL COMPARISON BETWEEN CYTOLOGY AND ANAL BIOPSY IN MEN WHO HAVE SEX WITH \\ MEN WHO ARE HIV CARRIERS AND ANAL INTRAEPITHELIAL NEOPLASIA ASSOCIATED WITH HPV \\ CONCORDÂNCIA HISTOPATOLÓGICA ENTRE A CITOLOGIA E A BIÓPSIA ANAL EM HOMENS QUE FAZEM SEXO \\ COM HOMENS PORTADORES DE HIV E NEOPLASIA INTRAEPITELIAL ASSOCIADAS COM HPV
}

\author{
CHRISTIAN BAJO RÍOS', FRANCISCO RAMÍREZ CABEZAS², RICARDO GARCÍA SOBARZO³ \\ ${ }^{1}$ Hospital General Regional №1 de Tijuana del Instituto Mexicano del Seguro Social, Tijuana, Baja California, México. \\ ${ }^{2}$ Hospital Universitario de Guayaquil del Ministerio de Salud Pública del Ecuador, hospital clínica San Francisco, Guayaquil, Ecuador. \\ ${ }^{3}$ ISSSTE, Instituto de Seguridad y Servicios Sociales de los Trabajadores del Estado, hospital Fray Junípero Serra, Tijuana, Baja California, México.
}

\begin{abstract}
Objetivo: determinar la concordancia de los resultados de la citología y la biopsia anal en hombres que tienen sexo con hombres (HSH) portadores de VIH y neoplasia intraepitelial anal (NIA) asociada a virus del papiloma humano (VPH). Materiales y métodos: estudio observacional transversal, de diciembre de 2011 a diciembre de 2013 para determinar la concordancia de los resultados de la citología y la biopsia anal en hombres que tienen sexo con hombres (HSH) portadores de VIH y NIA asociada a VPH en pacientes del hospital de Infectología del Centro Médico Nacional la Raza. Se realizó análisis estadísticos descriptivos. Resultados: se estudiaron 92 sujetos VIH positivos y VPH positivo, el reporte histopatológico definitivo de las biopsias anales fueron: neoplasia intraepitelial de bajo grado en $39 \%$, neoplasia intraepitelial de alto grado $4 \%$, células atípicas de significado incierto $3 \%$, normales en $27 \%$, con cambios inflamatorios reactivos en $23 \%$ y muestras inadecuadas para el diagnóstico el $9 \%$. La concordancia observada entre los dos métodos diagnósticos fue de 0.90 según alfa de Crobanch. Conclusión: el grado de concordancia del $90 \%$ de las biopsias y citologías anales, indica que son buenos métodos de seguimiento de los pacientes infectados con VIH y VPH, y permite detectar en forma oportuna lesiones precancerígenas, brindando diagnóstico y tratamiento oportuno.
\end{abstract}

PALABRAS CLAVE: neoplasias, carcinoma in situ, infección por Papillomavirus, VIH, conducta sexual.

Abstract

Objective: to determine the matching of the results of cytology and anal biopsy in men who have sex with men (MSM) who are HIV carriers and anal intraepithelial neoplasia (AIN) associated with human papillomavirus (HPV). Materials and methods: cross-sectional observational study from December 2011 to December 2013 to determine the matching of the results of cytology and anal biopsy in men who have sex with men (MSM) who are HIV carriers and AIN associated with HPV in patients with Infectious Diseases at the La Raza National Medical Center. Descriptive statistical analysis was performed. Results: 92 HIV positive and HPV positive subjects were studied, the final histopathological report of anal biopsies were: low-grade intraepithelial neoplasia in 39\%, high-grade intraepithelial neoplasia 4\%, atypical cells of undetermined significance 3\%, normal in 27, with reactive inflammatory changes in $23 \%$ and inadequate samples for diagnosis $9 \%$. The matching observed between both diagnostic methods was 0.90 based on Cronbach's alpha. Conclusion: the matching degree of $90 \%$ of the anal Pap smears and biopsies indicates that they are good monitoring methods of patients infected with HIV and HPV, and can detect precancerous lesions in a timely manner, providing prompt diagnosis and treatment.

KEYWORDS: neoplasms, carcinoma in situ, Papillomavirus infections, HIV, sexual behavior.

Resumo

Objetivo: determinar a concordância dos resultados do exame citológico e a biópsia anal em homens que fazem sexo com homens (HSH) portadores de HIV e neoplasia intraepitelial anal (NIA) associada com o vírus do papiloma humano (HPV). Material e método: estudo observacional transversal, de dezembro de 2011 a dezembro de 2013 para determinar a concordância dos resultados do exame de citologia e a biópsia anal em homens que fazem sexo com homens (HSH) portadores de HIV e NIA associadas com HPV em pacientes do hospital de Infectologia do Centro Médico Nacional da Raça. A análise estatística descritiva foi realizada. Resultados: foram estudados 92 indivíduos HIV positivos e HPV positivos, o laudo histopatológico definitivo de biópsias anais foi: neoplasia intra-epitelial de baixo grau em $39 \%$, neoplasia intraepitelial de alto grau 4\%, células atípicas de significado incerto $3 \%$, normal em $27 \%$, com alterações inflamatórias reativas em $23 \%$ e de amostragem inadequada para o diagnóstico em $9 \%$. A concordância observada entre os dois métodos de diagnóstico foi de 0,90 segundo alfa de Crobanch. Conclusão: 0 grau de concordância nos $90 \%$ de biópsias e citologias anais indica que são todos bons métodos de seguimento dos pacientes infectados com HIV e HPV e permite detectar oportunamente lesões pré-cancerosas, fornecendo o diagnóstico e o tratamento oportuno.

PALABRAS-CHAVE: neoplasias, carcinoma in situ, infecções por Papillomavirus, HIV, comportamento sexual. 


\section{INTRODUCCIÓN}

Aproximadamente 6230 casos nuevos de cáncer anal fueron reportados al final del año 2012 por la American Cáncer Society, y de éstos 780 ocasionaron directamente la muerte. ${ }^{1}$ La neoplasia intraepitelial anal (NIA) tiene un alto riesgo de convertirse en cáncer debido a que las lesiones preneoplásicas del conducto anal tienden a desarrollarse en la unión escamo columnar sujeta a trauma e infecciones recurrentes. ${ }^{2,3}$ En pacientes inmunocomprometidos es más frecuente que las lesiones intraepiteliales de alto grado del conducto anal tiendan a malignizarse. ${ }^{4}$

El departamento de sanidad del estado de Nueva York en Estados Unidos recomendó desde el año 2007 la realización de citología anal en la consulta inicial de estos pacientes, así como un seguimiento anual con anoscopía de alta resolución y biopsia cuando los resultados sean anormales. ${ }^{5}$

El agente causal de la NIA es el virus del papiloma humano (VPH); se han descubierto más de 100 tipos del virus, de los cuales 35 tienen afinidad por el epitelio ano genital. Éstos se han clasificado de forma general en dos grupos: los de bajo riesgo y los de alto riesgo. El $90 \%$ de las verrugas genitales son causadas por los tipos 6 y 11 , mientras que los tipos 16 y 18 se relacionan hasta en un $70 \%$ con cáncer cervical y anal. ${ }^{6} \mathrm{El}$ centro de control de enfermedades de los Estados Unidos (CDC) ha estimado que el 75 al $80 \%$ de los americanos con vida sexual activa contraerán la enfermedad en algún momento de su vida. ${ }^{7}$ En contraste con la prevalencia asociada a la edad de la infección por VPH del cérvix en mujeres, que disminuye a partir de los 30 años, la prevalencia de la infección por VPH del conducto anal en $\mathrm{HSH}$ y VIH positivos es alta; significa un 50 a 60 por ciento y permanece constante durante toda la vida. ${ }^{8,9}$ Esto podría estar relacionado a la infección por nuevos tipos de VPH resultado del gran número de parejas sexuales durante la vida de los pacientes $\mathrm{HSH}$.

Es por esto que el propósito de este trabajo fue determinar la concordancia de los resultados de la citología y la biopsia anal en HSH portadores de VIH y NIA asociada a VPH en pacientes del hospital de infectología del Centro Médico Nacional la Raza en México DF, y de este modo determinarla prevalencia deneoplasiaintraanal diagnosticada por biopsia anal y por citología en hombres que tienen sexo con hombres.

\section{MATERIALES Y MÉTODOS}

Se realizó un estudio descriptivo, observacional, transversal, en los servicios de Coloproctología, Anatomía Patológica y consulta externa del servicio de Infectología y el archivo clínico del Centro Médico Nacional la Raza de diciembre de 2011 a diciembre de 2013.

Los criterios de inclusión para la población seleccionada fueron: pacientes hombres que tienen sexo con hombres VIH positivo, mayores de 18 años, a los que se le realizó citología y biopsia anal. Se excluyó a los pacientes que no deseaban participar en el estudio ni realizarse la citología o biopsia, y a aquellos que no pudieron tomárseles las muestras.

Las variables que se estudiaron fueron: edad, tiempo con diagnóstico, número de parejas sexuales, tratamiento retroviral para VIH, conteo de CD4+ (inicial y actual), carga viral de RNA HIV (inicial y actual), resultados de la biopsia y de la citología.

Los sujetos elegibles llenaron la hoja de recolección de datos y se procedió a realizar la citología del conducto anal. No se emplearon lubricantes antes de la misma; se insertó un cepillo, de dacron o citobrush, en el conducto anal aproximadamente 3 a 4 centímetros para asegurar una toma adecuada de células de la zona de transición. Se abarcó toda la circunferencia del conducto anal realizando una presión gentil y una cuenta de diez segundos hasta su retiro, se efectuó la aplicación celular en las laminillas y éstas se conservaron en alcohol.

En relación a la toma de biopsia se insertó el anoscopio con la ayuda de un lubricante de base acuosa. A continuación, se introdujo un escobillón con una gasa empapada de ácido acético al $3 \%$, se dejó actuar por 2 minutos, a la espera de que la solución empape la pared del recto. Al introducir el anoscopio nuevamente se observaron las zonas que presentaron reacción aceto blanca positiva; este tejido sospechoso fue sometido a biopsia.

En caso de no encontrar lesiones sospechosas, se procedió a tomar biopsia de los cuadrantes de la zona de transición, preservando las muestras en formol. Se enviaron las biopsias a estudio histopatológico, y se compararán resultados entre sí. Los resultados se registraron en una base de datos para su posterior análisis estadístico. 
Se realizó un análisis descriptivo utilizando frecuencias y porcentajes o medianas con rangos intercuartiles (IQR) según fuera adecuado. Se consideró el intervalo de confianza del $95 \%$. Se utilizó la alfa de Cronbach para obtener el grado de concordancia que existe entre PAP y la biopsia para identificar NIA en los HSH portadores de VIH. Para el análisis de los datos se utilizó el software SPSS (IBM Corporation, EEUU) ${ }^{\circledR}$

RESULTADOS

Se incluyeron en total 92 pacientes que fueron referidos de la clínica de VIH del hospital de infectología del Centro Médico Nacional La Raza a la consulta externa de Coloproctología donde se realizó la citología y la biopsia anal. Un paciente perdió el seguro social durante el estudio y su expediente clínico no pudo ser valorado en el archivo por lo que fue excluido del análisis estadístico. La mediana de edad fue de 34 años (IQR 28-44), el tiempo de diagnóstico del VIH tuvo una mediana de 2 años (IQR 1-4), la mediana de número de parejas sexuales fue de 15 (IQR 6-30). Un total de $92 \%$ de los pacientes se encuentran bajo tratamiento antirretroviral altamente activo y $23 \%$ de ellos con inhibidores de la proteasa. Se obtuvo la mediana de CD4+ iniciales de los pacientes al momento del diagnóstico que fue de 241 células/uL (IQR 96442 ), el $45 \%$ de los pacientes presentaron CD4+ iniciales $<200$ células/uL. La mediana de carga viral de RNA VIH-1 al momento del diagnóstico fue 124,013 copias/ml (IQR 32,218-398,749), 59\% de los pacientes presentaron una carga viral de RNA VIH-1 >100,000 copias/ml (tabla 1 y 2).

TABLA 1. CARACTERÍSTICAS BASALES DE LA POBLACIÓN

\begin{tabular}{lrr}
\hline CARACTERÍSTICAS & MEDIANA (n=92) & RANGOS (IOR 25-75) \\
\hline Edad & 34 & $28-44$ \\
Tiempo de diagnóstico del VIH & 2 & $1-4$ \\
Número de parejas sexuales & 15 & $6-30$ \\
CD4+anual & $386 \mathrm{cel} / \mathrm{ml}$ & $275-572$ \\
Carga viral RNA VIH-1 actual & $36 \mathrm{copias} / \mathrm{ml}$ & $<20-827$ \\
CD4+inicial & $241 \mathrm{cel} / \mathrm{ml}$ & $96-442$ \\
Carga viral RNA VIH-1 inicial & $124.013 \mathrm{copias} / \mathrm{ml}$ & $32.218-398.749$ \\
\hline
\end{tabular}

TABLA 2. CARACTERÍSTICAS VIROLÓGICAS E INMUNOLÓGICAS EN LA POBLACIÓN

\begin{tabular}{lr}
\hline CARACTERÍSTICAS & PORCENTAJE \% ( $\mathbf{n = 9 2 )}$ \\
\hline CD4+actual $<200 \mathrm{cel} / \mu \mathrm{l}$ & 17 \\
CD4+inicial menor a $200 \mathrm{cel} / \mathrm{ml}$ & 45 \\
\hline Carga viral RNA VIH- 1 actual $>100,000$ copias $/ \mathrm{ml}$ & 7 \\
\hline Carga viral RNA VIH-1 actual $>100,000$ copias $/ \mathrm{ml}$ & 59 \\
TARAA & 92 \\
\hline Inhibidores de proteasa & 23 \\
\hline
\end{tabular}

Los resultados de las biopsias anales fueron los siguientes: neoplasia intraepitelial de bajo grado en $39 \%$ de los pacientes, neoplasia intraepitelial de alto grado $4 \%$, células atípicas de significado incierto $3 \%$, normales en $27 \%$, con cambios inflamatorios reactivos en $23 \%$ y muestras inadecuadas para el diagnóstico, el $9 \%$. Se encontró un $3 \%$ de lesiones asociadas a herpes virus y $1 \%$ con lesiones asociadas a treponema. (tabla 3). La frecuencia de neoplasia intraepitelial anal fue de $43 \%$ (95\% IC 25-45).

TABLA 3. ALTERACIONES HISTOPATOLÓGICAS EN LA BIOPSIA ANAL

\begin{tabular}{|lr|}
\hline CARACTERísticAS & PORCENTAJE \% (n=92) \\
\hline Citología normal & 27 \\
\hline Cambios reactivos inflamatorios & 23 \\
\hline Células atípicas significado incierto & 3 \\
\hline Neoplasia intraepitelial bajo grado & 39 \\
\hline Neoplasia intraepitelial alto grado & 4 \\
\hline Lesiones asociadas a herpes & 3 \\
\hline Lesiones asociadas a treponema & 1 \\
\hline Muestra inadecuada & 9 \\
\hline ALTERACIONES HISTOPATOLÓGICAS EN LA CITOLOGÍA ANAL \\
\hline Citología normal & 23 \\
\hline Cambios reactivos inflamatorios & 20 \\
\hline Células atípicas significado incierto & 3 \\
\hline Neoplasia intraepitelial bajo grado & 29 \\
\hline Neoplasia intraepitelial alto grado & 7 \\
\hline Muestra inadecuada & 12 \\
\hline
\end{tabular}

Los resultados de las citologías fueron los siguientes: neoplasia intraepitelial de bajo grado en $29 \%$ de los pacientes, $7 \%$ neoplasia intraepitelial de alto grado, células atípicas de significado incierto $3 \%$, citologías normales en $23 \%$, con cambios inflamatorios reactivos en $20 \%$ y muestras inadecuadas para el diagnóstico del $12 \%$. Se calculó la prevalencia de neoplasia intraepitelial anal con un nivel de confianza del $95 \%$ con un tamaño de la muestra de 92, 32 positivos con un intervalo de confianza del $95 \%$ dando como resultado $36 \%$ (95\% IC 25-45). La concordancia observada entre los dos métodos diagnósticos fue de 0.90 según alfa de Crobanch (tabla 4).

TABLA 4. CONCORDANCIA DE NIA ENTRE CITOLOGÍA Y BIOPSIA ANAL

\begin{tabular}{|l|r|r|r|}
\hline \multicolumn{4}{|c|}{ BIOPSIA } \\
\hline Citología & NIA presente & NIA ausente & Total \\
\hline NIA presente & 39 & 58 & 97 \\
\hline NIA ausente & 53 & 34 & 87 \\
\hline Total & 92 & 92 & 184 \\
\hline
\end{tabular}


DISCUSIÓN

Los hallazgos en este estudio son congruentes con lo publicado en la literatura médica internacional en donde se comunica que la prevalencia de NIA varía de un 46 a un 86 por ciento, debido a que los métodos diagnósticos, las poblaciones y los diseños de los estudios varían. ${ }^{10-13}$

Estos resultados son diferentes a los de Palefsky y colaboradores que en 1968, encontraron una prevalencia de $93 \%$ de DNA del VPH en pacientes con VIH hombres que tienen sexo con hombres mediante estudio de reacción en cadena de la polimerasa; sin embargo no reportaron la frecuencia de NIA asociada al virus. ${ }^{13}$

La fuerte correlación encontrada entre la citología y anoscopía suma evidencia a los resultados reportados por Mallari y colaboradores en su estudio retrospectivo de correlación entre citología y anoscopía en 285 sujetos de alto riesgo, cuya frecuencia fue del $66.5 \%$ de displasia co- $^{-}$ rroborada por biopsia, de ellas 42 presentaron neoplasia intraepitelial de alto grado.

Por citología detectaron una frecuencia del $19,5 \%$, la limitante de este estudio es que el análisis histopatológico se hizo por 2 diferentes laboratorios. ${ }^{10,14}$ Mientras que la fortaleza del estudio fue la realización de anoscopía de alta resolución además de citología, lo que podría explicar la elevada frecuencia encontrada en este estudio.

Las diferencias de los resultados encontrados en este estudio comparados con otros, es que no se cuenta con anoscopía de alta resolución, que en conjunto con citología y toma de biopsia mejoran la sensibilidad y especificidad diagnóstica porque permite visualizar cambios citológicos y vasculares relacionados con el VPH.

El beneficio de la detección oportuna en hombres que tienen sexo con hombres se ha demostrado en términos de costo-beneficio, realizando pruebas de detección oportuna anual para pacientes VIH+ y cada 2 a 3 años para el resto de la población. ${ }^{10,15}$ En los enfermos que resulten con displasia de alto grado algunos autores sugieren que solamente se deben vigilar cada 6 meses mediante una citología y anoscopia de alta resolución; los otros recomiendan el tratamiento quirúrgico con resección transanal de la lesión. ${ }^{13}$
En los hombres con VIH y que practican coito anal con hombres está demostrado el desarrollo del cáncer anal; pero la asociación del VIH con la progresión del cáncer anal es difícil de comprobar. ${ }^{9,16} \mathrm{El} \mathrm{VPH}$ es una infección asintomática que lleva al desarrollo de cáncer en una minoría de los pacientes que sufren esta infección por largo tiempo. Informes recientes indican que los sujetos con VIH+ viven mayor tiempo gracias a la terapia antirretroviral y tienen un aumento en la incidencia del cáncer anal.9,10 Este incremento de displasia y cáncer anal se ha observado en los pacientes VIH+ que tienen relaciones hombre con hombre, en los pacientes $\mathrm{VIH}+$ heterosexuales y en las mujeres $\mathrm{VIH}+$ que niegan relaciones anales. Los pacientes $\mathrm{VIH}+$ son más susceptibles de presentar displasia de bajo o alto grado y de progresar a alto grado en un período de 2 años. ${ }^{9,17,18}$ Estos dos hallazgos se presentan más frecuentemente cuando la cuenta de CD4 es < 200 células/mL.

Lo anterior sugiere una correlación estrecha de VIH y cáncer anal. Se sigue acumulando evidencia de que mientras los hombres y mujeres VIH+ vivan un tiempo mayor gracias a la terapia antirretroviral, la infección por VPH resultará en displasia o cáncer anal, y los pacientes más susceptibles serán los que estén más inmunocomprometidos. ${ }^{10,13,19}$

Cuando se comparan los resultados de la citología anal con los obtenidos mediante la biopsia, se encuentra una sensibilidad del 90 \% para el diagnóstico de lesiones de bajo y alto grado, así como de alteraciones inflamatorias. Sin embargo, la citología podría ser buen método de detección oportuna ya que es eficaz para la identificación de este tipo de patología, y además es muy barato y de fácil acceso para la población.

Cuando se comparan los resultados de la biopsia con los de la anoscopía de alta definición, se pueden observar diferencias para el diagnóstico de la lesión de bajo grado y de las alteraciones inflamatorias. Un inconveniente de la anoscopía de alta definición es que puede ser operador dependiente, al igual que la citología y la interpretación de la biopsia. Es importante señalar que las lesiones de alto grado son las que más se relacionan con la progresión hacia carcinoma epidermoide requiriendo tratamiento. 
Por ello, se propone con este estudio realizar citología anal en todos los casos, y en aquellos con lesiones de bajo o alto grado, continuar con anoscopía de alta definición y biopsia de las lesiones sospechosas. Si se corrobora la presencia de lesión de alto grado, el paciente deberá ser sometido a alguna de las diversas formas de tratamiento y continuar con el monitoreo a los 3 meses. En caso de ser negativo se sugiere continuar con la detección anual.

\section{CONCLUSIONES}

El grado de concordancia entre la biopsia y citología anal es elevada permitiendo realizar buenos seguimientos con ambos métodos. Ambas pruebas pueden detectar en forma oportuna lesiones precancerígenas, brindando un diagnóstico y tratamiento oportuno. Es necesario el uso de anoscopía de alta resolución y capacitación del personal para mejorar el porcentaje de toma de biopsias adecuadas.

\section{REFERENCIAS BIBLIOGRÁFICAS}

1. American Cancer Society. American Cancer Society. American Cancer Society. 2012.

2. Salit I, Tinmouth J, Lytwyn A. Anal Cancer screening: test characteristics of cytology and oncogenic HPV testing for the detection of anal dysplasia. The 12th Annual Conference on Retroviruses and Opportunistic infections (CROI); 2005; Boston, Massachussets.

3. Fenger C, Frisch M, Marti M, Parc R. Tumors of the anal canal. En: Pathology and Genetics of Tumours of the Digestive System. Lyon: IARC Press; 2010. p. 147-55.

4. Papaconstantinou HT, Lee AJ, Simmang CL, Ashfaq R, Gokaslan ST, Sokol S, et al. Screening methods for highgrade dysplasia in patients with anal condyloma. J Surg Res. el 1 de julio de 2005;127(1):8-13.

5. New York State Department of Health AIDS Institute. Neoplastic Complications of HIV Infection. New York, NY: New York State Department of Health AIDS Institute; 2011.

6. Palefsky JM, Rubin M. The epidemiology of anal human papillomavirus and related neoplasia. Obstet Gynecol Clin North Am. marzo de 2009;36(1):187-200.

7. Centers for Diseases Control and Prevention (CDC). 2008 Sexually Transmitted Diseases Surveillance. Atlanta, Georgia: Centers for Diseases Control and Prevention (CDC); 2008

8. Durante AJ, Williams AB, Da Costa M, Darragh TM, Khoshnood K, Palefsky JM. Incidence of anal cytological abnormalities in a cohort of human immunodeficiency virus-infected women. Cancer Epidemiol Biomark Prev Publ Am Assoc Cancer Res Cosponsored Am Soc Prev Oncol. julio de 2003;12(7):638-42.
9. Chin-Hong PV, Vittinghoff E, Cranston RD, Browne L, Buchbinder S, Colfax G, et al. Age-related prevalence of anal cancer precursors in homosexual men: the EXPLORE study. J Natl Cancer Inst. el 15 de junio de 2005;97(12):896-905.

10. Mallari AO, Schwartz TM, Luque AE, Polashenski PS, Rauh SM, Corales RB. Anal cancer screening in HIVinfected patients: is it time to screen them all? Dis Colon Rectum. diciembre de 2012;55(12):1244-50.

11. D’Souza G, Wiley DJ, Li X, Chmiel JS, Margolick JB, Cranston $\mathrm{RD}$, et al. Incidence and epidemiology of anal cancer in the multicenter AIDS cohort study. J Acquir Immune Defic Syndr 1999. el 1 de agosto de 2008;48(4):491-9.

12. Diamond C, Taylor TH, Aboumrad T, Bringman D, Anton-Culver $\mathrm{H}$. Increased incidence of squamous cell anal cancer among men with AIDS in the era of highly active antiretroviral therapy. Sex Transm Dis. mayo de 2005;32(5):314-20.

13. Palefsky JM, Holly EA, Ralston ML, Jay N. Prevalence and risk factors for human papillomavirus infection of the anal canal in human immunodeficiency virus (HIV)positive and HIV-negative homosexual men. J Infect Dis. febrero de 1998;177(2):361-7.

14. Echenique I, Phillips BR. Anal Warts and Anal Intradermal Neoplasia. Clin Colon Rectal Surg. marzo de 2011;24(1):31-8.

15. Fenger C, Nielsen VT. Dysplastic Changes in the Anal Canal Epithelium in Minor Surgical Specimens. Acta Pathol Microbiol Scand [A]. el 1 de marzo de 1981;89A(16):463-5.

16. Goldstone SE, Moshier E. Detection of oncogenic human papillomavirus impacts anal screening guidelines in men who have sex with men. Dis Colon Rectum. agosto de 2010;53(8):1135-42.

17. Schelhaas M, Ewers H, Rajamäki M-L, Day PM, Schiller JT, Helenius A. Human Papillomavirus Type 16 Entry: Retrograde Cell Surface Transport along Actin-Rich Protrusions. PLoS Pathog [Internet]. septiembre de 2008 [citado el 21 de diciembre de 2015];4(9). Recuperado a partir de: http://www.ncbi.nlm.nih.gov/pmc/articles/ PMC2518865

18. Schiller JT, Day PM, Kines RC. Current understanding of the mechanism of HPV infection. Gynecol Oncol. junio de 2010;118(1 Suppl):S12-7.

19. Cranston RD, Hart SD, Gornbein JA, Hirschowitz SL, Cortina G, Moe AA. The prevalence, and predictive value, of abnormal anal cytology to diagnose anal dysplasia in a population of HIV-positive men who have sex with men. Int J STD AIDS. febrero de 2007;18(2):77-80. 